\title{
WISEP J180026.60+013453.1: A Nearby Late L Dwarf Near the Galactic Plane
}

\author{
John E. Gizis \\ Department of Physics and Astronomy, University of Delaware, Newark, DE 19716, USA \\ Adam J. Burgasser \\ Center for Astrophysics and Space Science, University of California San Diego, La Jolla, \\ CA 92093, USA \\ Jacqueline K. Faherty ${ }^{1}$ \\ Department of Astrophysics, American Museum of Natural History, Central Park West at \\ 79th Street, New York, NY 10034, USA \\ Philip J. Castro \\ Department of Physics and Astronomy, University of Delaware, Newark, DE 19716, USA \\ Michael M. Shara ${ }^{1}$ \\ Department of Astrophysics, American Museum of Natural History, Central Park West at \\ 79th Street, New York, NY 10034, USA
}

\begin{abstract}
We report a nearby L7.5 dwarf discovered using the Preliminary Data Release of the Wide-field Infrared Survey Explorer (WISE) and the Two Micron All-Sky

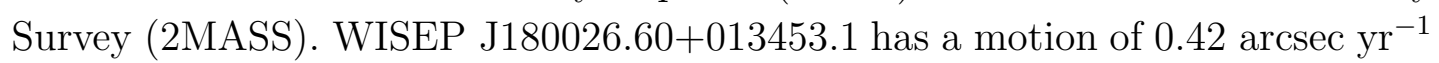
and an estimated distance of $8.8 \pm 1.0 \mathrm{pc}$. With this distance, it currently ranks as the sixth closest known L dwarf, although a trigonometric parallax is needed to confirm this distance. It was previously overlooked because it lies near the Galactic Plane $\left(b=12^{\circ}\right)$. As a relatively bright and nearby late $\mathrm{L}$ dwarf with normal near-infrared colors, W1800+0134 will serve as a benchmark for studies of cloud-related phenomena in cool substellar atmospheres.

Subject headings: brown dwarfs - infrared: stars - Proper motions - stars: individual: WISEP J180026.60+013453.1

\footnotetext{
${ }^{1}$ Visiting Astronomer at the Infrared Telescope Facility, which is operated by the University of Hawaii under Cooperative Agreement no. NNX-08AE38A with the National Aeronautics and Space Administration, Science Mission Directorate, Planetary Astronomy Program.
} 


\section{Introduction}

One of the main scientific goals of the Wide-field Infrared Survey Explorer (WISE) (Wright et al. 2010) is to identify the brown dwarfs and low-mass stars closest to the Sun. WISE has now surveyed the entire sky in four mid-infrared filters, with $\sim 58 \%$ of the sky included in the April 2011 Preliminary Data Release. Together with the three nearinfrared Two Micron All-Survey (2MASS) filters (Skrutskie et al. 2006), the peaks of the spectral energy distributions of any nearby brown dwarfs with $T_{\text {eff }} \gtrsim 300 \mathrm{~K}$ are well sampled (Mainzer et al. 2011).

Although hundreds of brown dwarfs with M, L, or T spectral types are now known, very few of them are closer than ten parsecs to the Sun. The nearest known L dwarfs with trigonometric parallaxes are the L8 dwarf DENIS-P J0255.0-4700 (Martín et al. 1999) at 4.97 \pm 0.10 pc (Costa et al. 2006), the L5 dwarf 2MASSW J1507476-162738 (Reid et al.|2000) at $7.33 \pm$ 0.03 pc (Dahn et al. 2002), the L5 dwarf 2MASSI J0835425-081923 (Cruz, Reid, Liebert, Kirkpatrick, \& Lo 2003 ) at $8.5 \pm 0.8 \mathrm{pc}$ (Andrei et al. 2011), the L3.5 2MASSW J0036159+182110 (Reid et al. 2000) at $8.76 \pm 0.06 \mathrm{pc}$ (Dahn et al. 2002), and the L5.5 dwarf 2MASS J17502484-0016151 (Kendall. Jones, Pinfield, Pokorny, Folkes, Weights, Jenkins, \& Mauron 2007) at $9.2 \pm 0.2$ (Andrei et al. 2011). The lithium M9 brown dwarf LP 944-20 (Tinney 1998), measured at $4.97 \pm 0.11$ pc (Tinney 1996), also deserves mention. Other apparently nearby L dwarfs still lack reliable trigonometric parallaxes. The "blue" L6 dwarf SDSS J141624.08+134826.7 (Bowler, Liu, \& Dupuy 2010a; Schmidt et al. 2010a) is thought to lie at $8.4 \pm 1.9$ or $8.0 \pm 1.6$ on the basis of photometry, while Scholz (2010) argue for a parallax distance of $7.9 \pm 1.7$ pc based on limited astrometry. Looper et al. (2008b) list five additional L dwarfs with estimated distances between 9.4 and $9.9 \mathrm{pc} 1$ Along with these warmer brown dwarfs, more than a dozen $\mathrm{T}$ dwarfs are known within ten parsecs. WISE, whose filters are ideally suited for finding cold late-type T dwarfs, is already adding to the census: Mainzer et al. (2011) report a late-T dwarf at $d \approx 6-10 \mathrm{pc}$, while Scholz et al. (2011) have identified two high proper motion, late-T dwarfs with $d \approx 5$ pc using WISE, 2MASS and SDSS.

L dwarfs are useful probes for studying atmospheric properties of objects below 2200K, where clouds and dust opacity are important to the observed spectral energy distribution. In addition to their intrinsic interest, the latest-type $\mathrm{L}$ dwarfs are potential analogs to massive hot exoplanets such as those found around HR 8799 (Bowler et al. 2010b; Marois et al. 2010; Barman et al. 2011). Because they are isolated in the field rather than found orbiting a

\footnotetext{
${ }^{1}$ We follow Burgasser et al. (2008) in viewing 2MASS J11263991-5003550 as a blue L4.5 at 15 pc rather than a blue L9 at 8 pc (Folkes et al. 2007). Henrv et al. (2006) have shown by trigonometric parallax that the GJ 1001 (LHS 102) system (Goldman et al. 1999) lies beyond 10 parsecs.
} 
nearby bright star they can be studied and characterized in great detail making them vital test beds for refinement.

We have initiated an effort to detect high proper motion stars by comparing the WISE and 2MASS surveys. Here, we report the identification of a bona-fide, nearby late-L brown dwarf, WISEP J180026.60+013453, as a result of this effort. We present our observations and data analysis in Section 2, We discuss the possibility of binarity in Section 3 ,

\section{Identification and Observations}

\subsection{WISE Selection}

We selected WISE sources with $|b|>10$ that were detected at W1 $(3.4 \mu \mathrm{m}), \mathrm{W} 2(4.6 \mu \mathrm{m})$ and W3 $(12 \mu \mathrm{m})$ with $\mathrm{W} 1<12$ but had no 2MASS counterpart within 3 arcseconds according to the WISE Preliminary Source Catalog. All stars or warm brown dwarfs with W1< 12 should have 2MASS detections at $\mathrm{K}_{s}$ band. The WISE, 2MASS, and photographic (Digitized Sky Survey, DSS) images were examined by eye to look for proper motion candidates. Almost all stars have $\mathrm{W} 1-\mathrm{W} 2<0.3$ and $\mathrm{W} 1-\mathrm{W} 3<0.7$, but we noted a number of candidate proper motion objects with redder W1-W2 colors. Most of these turned out to be known late-M or L dwarfs, but the source WISEP J180026.60+013453.1 (Table1) was previously unrecognized. As seen in Figure 1, this WISE source may be paired with 2MASS J18002648+0134565 $\left(\mathrm{J}=14.30 \pm 0.04, \mathrm{H}=13.12 \pm 0.04, \mathrm{~K}_{s}=12.42 \pm 0.03\right)$, which otherwise would not have a WISE counterpart. We hereafter abbreviate the source name as W1800+0134. The object was likely overlooked previously because it lies near the Galactic Plane $\left(b=12^{\circ}\right)$, although the field is not very crowded.

The apparent proper motion from 2MASS to WISE is $\mu_{\alpha}=0.20 \pm 0.02 \operatorname{arcsec}_{\mathrm{yr}^{-1}}$ and $\mu_{\delta}=-0.36 \pm 0.02 \operatorname{arcsec} \mathrm{yr}^{-1}$. This is relative to other stars in the image, with uncertainties based on the uncertainties of the WISE and 2MASS astrometry. However, because the observations were taken in 2010 March 20-23 (WISE) and 2000 September 23 (2MASS), parallax will affect the observed positions. Using the NOVAS V3.0 software (Kaplan et al. 2009), we find that the offset in RA is 230 mas if W1800+0134 is 8.8 pc away, as estimated later. Applying this correction, we find $\mu_{R A}=0.22 \pm 0.02 \operatorname{arcsec}_{\mathrm{yr}^{-1}}$ and $\mu_{D E C}=-0.36 \pm 0.02 \operatorname{arcsec} \mathrm{yr}^{-1}$. A possible very faint source is seen at the predicted position on the infrared (I-band) Second Palomar Sky Survey plate (Reid et al. 1991), but as expected for an L dwarf, it is not detected on the blue or red photographic plates (Figure 1,) We also identify W1800+0134 with DENIS J180026.4+013457, $\mathrm{J}=14.07 \pm 0.10$ and $\mathrm{K}=12.45 \pm 0.12$ in the 3rd release of the DENIS database. It was apparently not detected in the I band 
images, suggesting $\mathrm{I} \gtrsim 18$. Because the DENIS observation is close in time to the 2MASS observation but is lower signal-to-noise, the detection confirms that W1800 has moved but was not included in the proper motion determination.

\subsection{Near-Infrared Spectroscopy}

A low-resolution IRTF SpeX (Rayner et al. 2003) spectrum was obtained on 2011 June 22 (UT) and processed using SpexTool (Vacca, Cushing, \& Rayner 2003; Cushing, Vacca, \& Rayner 2004). Spectra were obtained in prism mode using the 0.8 " slit aligned at the parallactic angle. The resolution of the corresponding data spanning $0.7-2.5 \mu \mathrm{m}$ was $\lambda / \Delta \lambda \approx 90$. Conditions were clear and the seeing was 0.7 " at K. We obtained 4 individual exposure times of 120s in an ABBA dither pattern along the slit. Immediately after the science observations we observed the A0V star HD 171149 at a similar airmass for telluric corrections and flux calibration. Internal flat-field and Ar arc lamp exposures were acquired for pixel response and wavelength calibration, respectively.

Our spectrum is shown in Figure 2. We fit the spectrum to L and T dwarf templates also observed with SpeX 2 The best matching template is 2MASS J02572581-3105523, classified as L8 in the optical by Kirkpatrick et al. (2008) on the Kirkpatrick et al. (1999) system and observed with SpeX by Siegler et al. (2007). The indices and spectral type relations defined by Burgasser (2007) give L6.5 \pm 0.07 (H2O-J), L5.8 $\pm 0.07(\mathrm{H} 2 \mathrm{O}-\mathrm{H})$, L6.9 \pm 0.09 (H2O$\mathrm{K})$, and $\mathrm{L} 7.7 \pm 0.04(\mathrm{CH} 4-\mathrm{K})$, for a mean classification of $\mathrm{L} 7 \pm 1$. The scatter is typical for near-infrared spectral typing of L dwarfs (e.g., Burgasser 2007; Stephens et al. 2009). Combining the index type with the best-fit template type, we adopt a near-infrared spectral classification of L7.5 \pm 0.5 for $\mathrm{W} 1800+0134$.

Using the Looper et al. (2008a) spectral type-absolute magnitude relationships, and propagating uncertainties through Monte Carlo analysis, we estimate a distance of $8.8 \pm 1.0$ pc from the 2MASS $\mathrm{J}$ and $\mathrm{K}_{s}$ photometry. Comparison of this estimated distance to the $\mathrm{L}$ dwarf distances listed in Section 1 suggests W1800+0134 ranks as the sixth closest known L dwarf, but even a one parsec change could move it up to third or down to eighth or later. In any case, a trigonometric parallax is needed to realize W1800+0134's scientific potential as one of the closest, brightest late-L dwarfs for detailed study.

\footnotetext{
${ }^{2}$ Low-resolution $\mathrm{L}$ and $\mathrm{T}$ dwarf templates were drawn from the SpeX Prism Spectral Libraries, http: //www.browndwarfs.org/spexprism
} 


\subsection{Physical Properties}

The Looper et al. (2008a) polynomial fits to the Golimowski et al. (2004) temperature scale indicate that $T_{\text {eff }}=1490 \mathrm{~K}$ and $B C_{K}=3.24$ for L7.5 dwarfs. With our estimated distance, W1800+0134 is then $\log L / L_{\odot} \approx-4.5 \pm 0.3$. The Stephens et al. (2009) relations, also based on a reanalysis of Golimowski et al. (2004), make them somewhat cooler at $T_{\text {eff }}=$ $1430 \pm 100 \mathrm{~K}$. The tangential velocity $\left(18 \pm 2 \mathrm{~km} \mathrm{~s}^{-1}\right)$ is below the median for late $\mathrm{L}$ dwarfs (Faherty et al. 2009). According to the Burrows et al. (1997) models, for ages of 0.5, 1, and 5 Gyr, the mass of a $T_{e f f}=1430 \mathrm{~K}$ dwarf is $0.04 M_{\odot}, 0.05 M_{\odot}$, and $0.074 M_{\odot}$ respectively; the Chabrier et al. (2000) models give similar results. For all ages, it is below the hydrogenburning limit. An optical spectrum could determine whether W1800+0134 is above or below the lithium-burning limit (Magazzu, Martin, \& Rebolo 1993; Kirkpatrick et al. 2000).

\section{Is W1800+0134 a Binary?}

Some $\sim 20 \%$ of very-low-mass binaries in magnitude-limited samples are typically resolved as doubles (Gizis et al. 2003; Bouv et al. 2003; Burgasser et al. 2006). If W1800+0134 were a near-equal luminosity double, the estimated distance would increase to $\sim 12 \mathrm{pc}$. The low-resolution spectrum shows some evidence of structure in the H-band around the region of the $1.6 \mu \mathrm{m} \mathrm{CH}$ band, but no indication of $\mathrm{CH}_{4}$ at K-band (see Figure 2.) Unresolved L dwarf plus $\mathrm{T}$ dwarf binary spectra frequently show this pattern of $\mathrm{CH}_{4}$ onset in the nearinfrared (Cruz et al. 2004; Burgasser 2007). To investigate this possibility, we compared the SpeX spectrum to combinations of $\mathrm{L}$ and $\mathrm{T}$ dwarf binary templates from the SpeX Prism Spectral Libraries, following the procedures described in Burgasser (2007). The best fit binary has the same $\chi^{2}$ residual as the best fit single source; as such, there is no statistically robust evidence of $\mathrm{W} 1800+0134$ being an L plus T dwarf binary.

The 2MASS $\mathrm{J}_{-} \mathrm{K}_{s}=1.88 \pm 0.05$ color is neither unusually blue nor red compared to other L7-L8 dwarfs (Faherty et al. 2009; Schmidt et al. 2010b). The DENIS non-detection at I-band (corresponding to $I-J \gtrsim 3.7)$ is also consistent with the observed $(I-J \approx 4)$ colors of other L7-L8 dwarfs (Phan-Bao et al. 2008). To assess whether the WISE colors are typical, we have searched for known objects with an L7, L7.5, or L8 classification in the WISE Preliminary database with uncertainties less than 0.1 in W1 and W2 and 0.2 in W3. The seven matching dwarfs are listed in Table 2, The observed mean and standard deviations of the apparently single objects are $\mathrm{W} 1-\mathrm{W} 2=0.54 \pm 0.05$ and $\mathrm{W} 2-\mathrm{W} 3=1.01 \pm 0.09$, consistent with W1800+0134's colors $(0.50 \pm 0.06,1.16 \pm 0.08)$. The doubles SDSSp J042348.57-041403.5 and 2MASS J05185995-2828372 are somewhat redder but the uncertainties in $\mathrm{W}_{3}$ are large (0.08 and 0.17 respectively.) 
We note for completeness that we found no other common proper motion companions within 30 arcminutes of W1800+0134, in WISE or 2MASS, ruling out widely-separated main sequence to late- $\mathrm{T}$ brown dwarf companions to a projected separation of 15,000 AU.

\section{Conclusion}

We have identified a previously overlooked bright and nearby L7.5 dwarf, near the Galactic Plane. Together with the recent discoveries of the M9 DENIS-P J115927.4-524718 at 9.6 pc $\left(b=9^{\circ}\right.$; Phan-Bao et al. 2008), the T6 DENIS J081730.0-615520 at $4.9 \mathrm{pc}\left(b=-14^{\circ}\right.$; Artigau et al. 2010), the T9 UGPS J072227.51-054031.2 at $4.1 \mathrm{pc}\left(b=4^{\circ}\right.$; Lucas et al. 2010), and the T9 WISEPC J045853.90+643451.9 at 6-10 pc $\left(b=13^{\circ}\right.$; Mainzer et al. 2011), it is clear that multi-color, multi-survey searches for nearby brown dwarfs in the Galactic Plane region are now feasible. Such objects are important because their proximity enables more detailed studies of the physical properties of brown dwarfs, as well as any potential planetary companions. As a very bright late-L dwarf with normal colors, studies of W1800+0134's cloud properties through variability and polarization measurements (e.g., Artigau et al.|2009; Goldman et al.|2009), and rotation through high-resolution spectroscopy (e.g., Zapatero Osorio et al. 2006), will be helpful in characterizing the structure and dynamics of cloudy atmospheres at the L/T transition, and will be relevant to exploring the cloud properties of hot exoplanets (Madhusudhan, Burrows, \& Currie 2011).

We thank the referee (Chris Tinney) for useful comments. This publication makes use of data products from the Wide-field Infrared Survey Explorer, which is a joint project of the University of California, Los Angeles, and the Jet Propulsion Laboratory/California Institute of Technology, funded by the National Aeronautics and Space Administration. This publication makes use of data products from the Two Micron All Sky Survey, which is a joint project of the University of Massachusetts and the Infrared Processing and Analysis Center/California Institute of Technology, funded by the National Aeronautics and Space Administration and the National Science Foundation. This research has made use of the NASA/ IPAC Infrared Science Archive, which is operated by the Jet Propulsion Laboratory, California Institute of Technology, under contract with NASA. This research has made use of the VizieR catalogue access tool, CDS, Strasbourg, France. This research has made use of the SIMBAD database, operated at CDS, Strasbourg, France. The Digitized Sky Surveys were produced at the Space Telescope Science Institute under U.S. Government grant NAG $\mathrm{W}-2166$. The images of these surveys are based on photographic data obtained using the Oschin Schmidt Telescope on Palomar Mountain and the UK Schmidt Telescope. The plates were processed into the present compressed digital form with the permission of these institu- 
tions. This research made use of APLpy, an open-source plotting package for Python hosted at http://aplpy.github.com. This research has benefitted from the M, L, and T dwarf compendium housed at DwarfArchives.org and maintained by Chris Gelino, Davy Kirkpatrick, and Adam Burgasser. This research has benefitted from the SpeX Prism Spectral Libraries, maintained by Adam Burgasser at http://www.browndwarfs.org/spexprism

\section{REFERENCES}

Andrei, A. H., Smart, R. L., Penna, J. L., et al. 2011, AJ, 141, 54

Artigau, É., Bouchard, S., Doyon, R., \& Lafrenière, D. 2009, ApJ, 701, 1534

Artigau, É., Radigan, J., Folkes, S., et al. 2010, ApJ, 718, L38

Barman, T. S., Macintosh, B., Konopacky, Q. M., \& Marois, C. 2011, ApJ, 733, 65

Bouy, H., Brandner, W., Martín, E. L., et al. 2003, AJ, 126, 1526

Bowler, B. P., Liu, M. C., \& Dupuy, T. J. 2010a, ApJ, 710, 45

Bowler, B. P., Liu, M. C., Dupuy, T. J., \& Cushing, M. C. 2010b, ApJ, 723, 850

Burgasser, A. J. 2007, ApJ, 659, 655

Burgasser, A. J., Kirkpatrick, J. D., Cruz, K. L., et al. 2006, ApJS, 166, 585

Burgasser, A. J., Looper, D. L., Kirkpatrick, J. D., et al. 2008, ApJ, 674, 451

Burrows, A., Marley, M., Hubbard, W. B., et al. 1997, ApJ, 491, 856

Chabrier, G., Baraffe, I., Allard, F., \& Hauschildt, P. 2000, ApJ, 542, 464

Chiu, K., Fan, X., Leggett, S. K., et al. 2006, AJ, 131, 2722

Costa, E., Méndez, R. A., Jao, W., et al. 2006, AJ, 132, 1234

Cruz, K. L., Burgasser, A. J., Reid, I. N., \& Liebert, J. 2004, ApJ, 604, L61

Cruz, K. L., Reid, I. N., Liebert, J., Kirkpatrick, J. D., \& Lowrance, P. J. 2003, AJ, 126, 2421

Cushing, M. C., Vacca, W. D., \& Rayner, J. T. 2004, PASP, 116, 362

Dahn, C. C., Harris, H. C., Vrba, F. J., et al. 2002, AJ, 124, 1170 
Faherty, J. K., Burgasser, A. J., Cruz, K. L., et al. 2009, AJ, 137, 1

Folkes, S. L., Pinfield, D. J., Kendall, T. R., \& Jones, H. R. A. 2007, MNRAS, 378, 901

Geballe, T. R., Knapp, G. R., Leggett, S. K., et al. 2002, ApJ, 564, 466

Gizis, J. E., Reid, I. N., Knapp, G. R., et al. 2003, AJ, 125, 3302

Goldman, B., Delfosse, X., Forveille, T., Afonso, C., Alard, C., Albert, J. N., Andersen, J., Ansari, R., et al. 1999, A\&A, 351, L5

Goldman, B., Pitann, J., Zapatero Osorio, M. R., et al. 2009, A\&A, 502, 929

Golimowski, D. A., Leggett, S. K., Marley, M. S., et al. 2004, AJ, 127, 3516

Henry, T. J., Jao, W., Subasavage, J. P., et al. 2006, AJ, 132, 2360

Kaplan, G., Bangert, J., Bartlett, J., Puatua, W., \& Monet, A. 2009, User's Guide to NOVAS 3.0, Vol. USNO Circular 180 (Washington, DC: USNO)

Kendall, T. R., Jones, H. R. A., Pinfield, D. J., et al. 2007, MNRAS, 374, 445

Kirkpatrick, J. D., Cruz, K. L., Barman, T. S., et al. 2008, ApJ, 689, 1295

Kirkpatrick, J. D., Reid, I. N., Liebert, J., et al. 1999, ApJ, 519, 802

Kirkpatrick, J. D., Reid, I. N., Liebert, J., et al. 2000, AJ, 120, 447

Looper, D. L., Gelino, C. R., Burgasser, A. J., \& Kirkpatrick, J. D. 2008a, ApJ, 685, 1183

Looper, D. L., Kirkpatrick, J. D., Cutri, R. M., et al. 2008b, ApJ, 686, 528

Lucas, P. W., Tinney, C. G., Burningham, B., et al. 2010, MNRAS, 408, L56

Madhusudhan, N., Burrows, A., \& Currie, T. 2011, ArXiv e-prints

Magazzu, A., Martin, E. L., \& Rebolo, R. 1993, ApJ, 404, L17

Mainzer, A., Cushing, M. C., Skrutskie, M., et al. 2011, ApJ, 726, 30

Marois, C., Zuckerman, B., Konopacky, Q. M., Macintosh, B., \& Barman, T. 2010, Nature, 468,1080

Martín, E. L., Delfosse, X., Basri, G., et al.1999, AJ, 118, 2466

Phan-Bao, N., Bessell, M. S., Martín, E. L., et al. 2008, MNRAS, 383, 831 
Rayner, J. T., Toomey, D. W., Onaka, P. M., et al. 2003, PASP, 115, 362

Reid, I. N., Brewer, C., Brucato, R. J., et al. 1991, PASP, 103, 661

Reid, I. N., Kirkpatrick, J. D., Gizis, J. E., et al. 2000, AJ, 119, 369

Schmidt, S. J., West, A. A., Burgasser, A. J., Bochanski, J. J., \& Hawley, S. L. 2010a, AJ, 139,1045

Schmidt, S. J., West, A. A., Hawley, S. L., \& Pineda, J. S. 2010b, AJ, 139, 1808

Scholz, R. 2010, A\&A, 510, L8+

Scholz, R. ., Bihain, G., Schnurr, O., \& Storm, J. 2011, ArXiv e-prints

Siegler, N., Close, L. M., Burgasser, A. J., et al. 2007, AJ, 133, 2320

Skrutskie, M. F., Cutri, R. M., Stiening, R., et al. 2006, AJ, 131, 1163

Stephens, D. C., Leggett, S. K., Cushing, M. C., et al. 2009, ApJ, 702, 154

Tinney, C. G. 1996, MNRAS, 281, 644

-. 1998, MNRAS, 296, L42+

Vacca, W. D., Cushing, M. C., \& Rayner, J. T. 2003, PASP, 115, 389

Wright, E. L., Eisenhardt, P. R. M., Mainzer, A. K., et al. 2010, AJ, 140, 1868

Zapatero Osorio, M. R., Martín, E. L., Bouy, H., et al. 2006, ApJ, 647, 1405

This preprint was prepared with the AAS IATEX macros v5.2. 
Table 1. WISEP J180026.60+013453.1

\begin{tabular}{lc}
\hline \hline \multicolumn{1}{c}{ Parameter } & W1800+0134 \\
\hline WISE RA(J2000) & 180026.60 \\
WISE Dec (J2000) & +013453.1 \\
WISE Epoch & 2010.22 \\
2MASS J [mag] & $14.30 \pm 0.04$ \\
2MASS H [mag] & $13.12 \pm 0.04$ \\
2MASS K $[$ [mag] & $12.42 \pm 0.03$ \\
WISE W1 $[\mathrm{mag}]$ & $11.53 \pm 0.05$ \\
WISE W2 [mag] & $11.03 \pm 0.04$ \\
WISE W3 [mag] & $9.88 \pm 0.07$ \\
WISE W4 [mag] & $>8.39$ \\
$\mu_{\alpha} \cos \delta(\mathrm{mas} / \mathrm{yr})$ & $220 \pm 20$ \\
$\mu_{\delta}(\mathrm{mas} / \mathrm{yr})$ & $-360 \pm 20$ \\
Sp Type $(\mathrm{Near}-\mathrm{IR})$ & $\mathrm{L} 7.5 \pm 0.5$ \\
distance $(\mathrm{pc})$ & $8.8 \pm 1.0$ \\
$v_{\text {tan }} \mathrm{km} \mathrm{s} \mathrm{s}^{-1}$ & $18 \pm 2$ \\
$T_{\text {eff }}(K)$ & $1430 \pm 100$ \\
$\log L / L_{\odot}$ & $-4.5 \pm 0.3$ \\
\hline
\end{tabular}

Table 2. WISE Data for L7-L8 dwarfs

\begin{tabular}{lcccccc}
\hline \hline \multicolumn{1}{c}{ Discovery Name } & WISEP & W1 & W1-W2 & W2-W3 & Double? & Ref. \\
\hline 2MASS J03185403-3421292 & J031854.37-342128.7 & 12.62 & 0.50 & $0.97 \pm 0.11$ & $\ldots$ & 1 \\
SDSSp J042348.57-041403.5 & J042348.32-041402.5 & 12.18 & 0.61 & $1.03 \pm 0.09$ & $\mathrm{Y}$ & 2,3 \\
2MASS J05185995-2828372 & J051859.89-282840.2 & 13.41 & 0.59 & $1.09 \pm 0.17$ & $\mathrm{Y}$ & 4,3 \\
2MASSI J0825196+211552 & $\mathrm{J} 082519.24+211548.5$ & 12.08 & 0.52 & $1.02 \pm 0.09$ & $\ldots$ & 5 \\
SDSSp J085758.45+570851.4 & $\mathrm{J} 085757.95+570847.5$ & 12.03 & 0.61 & $1.12 \pm 0.06$ & $\ldots$ & 2 \\
2MASSW J1632291+190441 & $\mathrm{J} 163229.39+190439.9$ & 13.14 & 0.52 & $0.92 \pm 0.19$ & $\ldots$ & 6 \\
2MASSW J1728114+394859 & $\mathrm{J} 172811.53+394859.1$ & 13.10 & 0.49 & $0.94 \pm 0.12$ & $\mathrm{Y}$ & 5,7 \\
WISEP J180026.60+013453.1 & $\mathrm{J} 180026.60+013453.1$ & 11.53 & 0.55 & $1.16 \pm 0.08$ & $\ldots$ & 8 \\
\hline
\end{tabular}

Note. - The uncertainty in $\mathrm{W}_{1}$ is 0.03 mags and in $\mathrm{W}_{1}-\mathrm{W}_{2}$ is 0.04 mags for all objects.

References. - 1. Kirkpatrick et al. (2008) 2. Geballe et al. (2002) 3. Burgasser et al. (2006) 4. Cruz et al. (2004) 5. Kirkpatrick et al. (2000) 6. Kirkpatrick et al. (1999) 7. Gizis et al. (2003) 8. This paper 


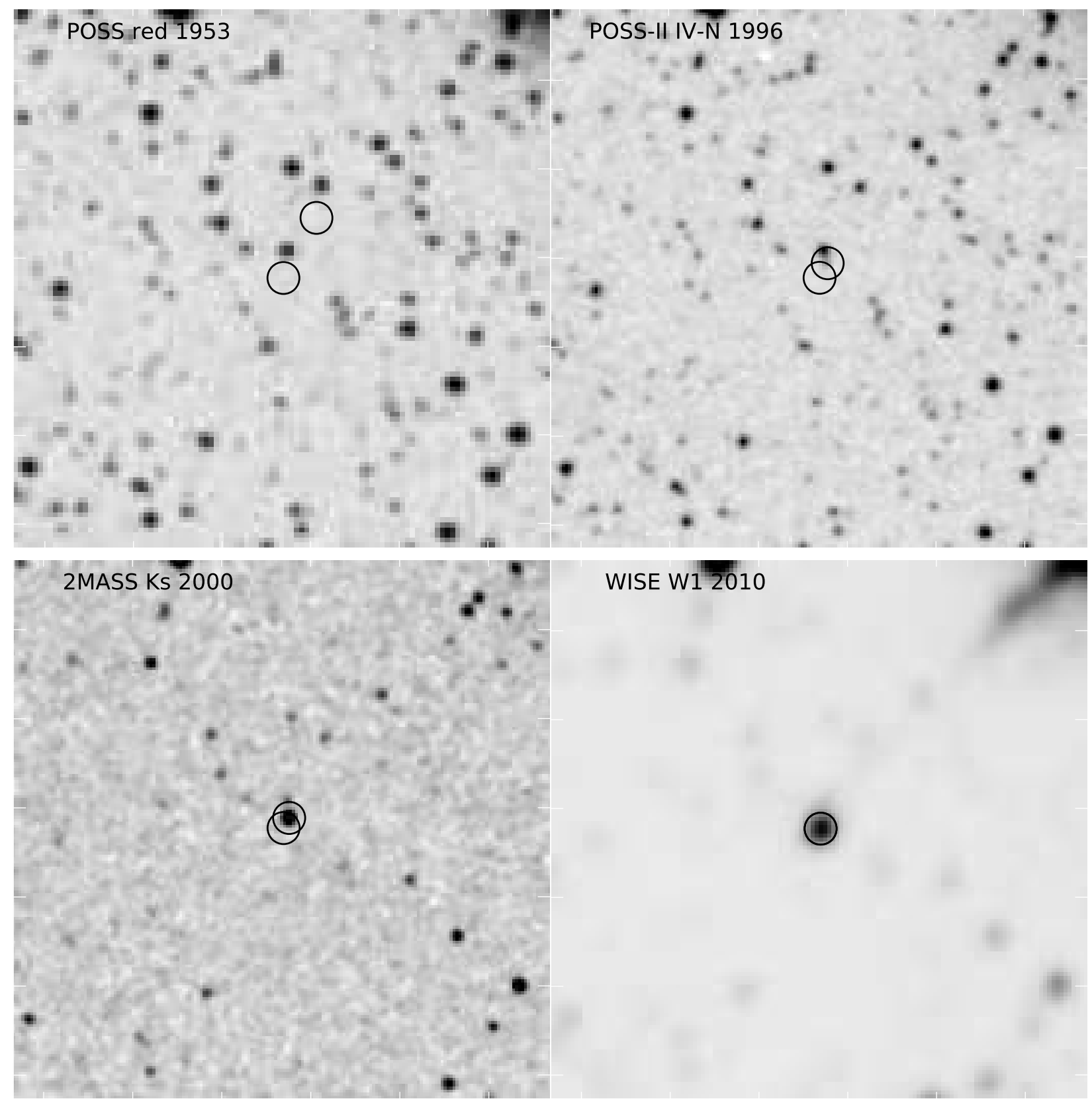

Fig. 1.- Finder charts for WISEP J180026.60+013453.1: Upper left, July 1953 Palomar Sky Survey (POSS) red plate. Upper right, July 1996 Second Palomar Sky Survey (POSSII) infrared plate. Lower left, September 2000 2MASS K band. Lower right, March 2010 WISE W1 band. In each case, the central circle marks the observed WISE position and an additional circle marks the expected position at that epoch. All are 3 arcminutes on a side. 


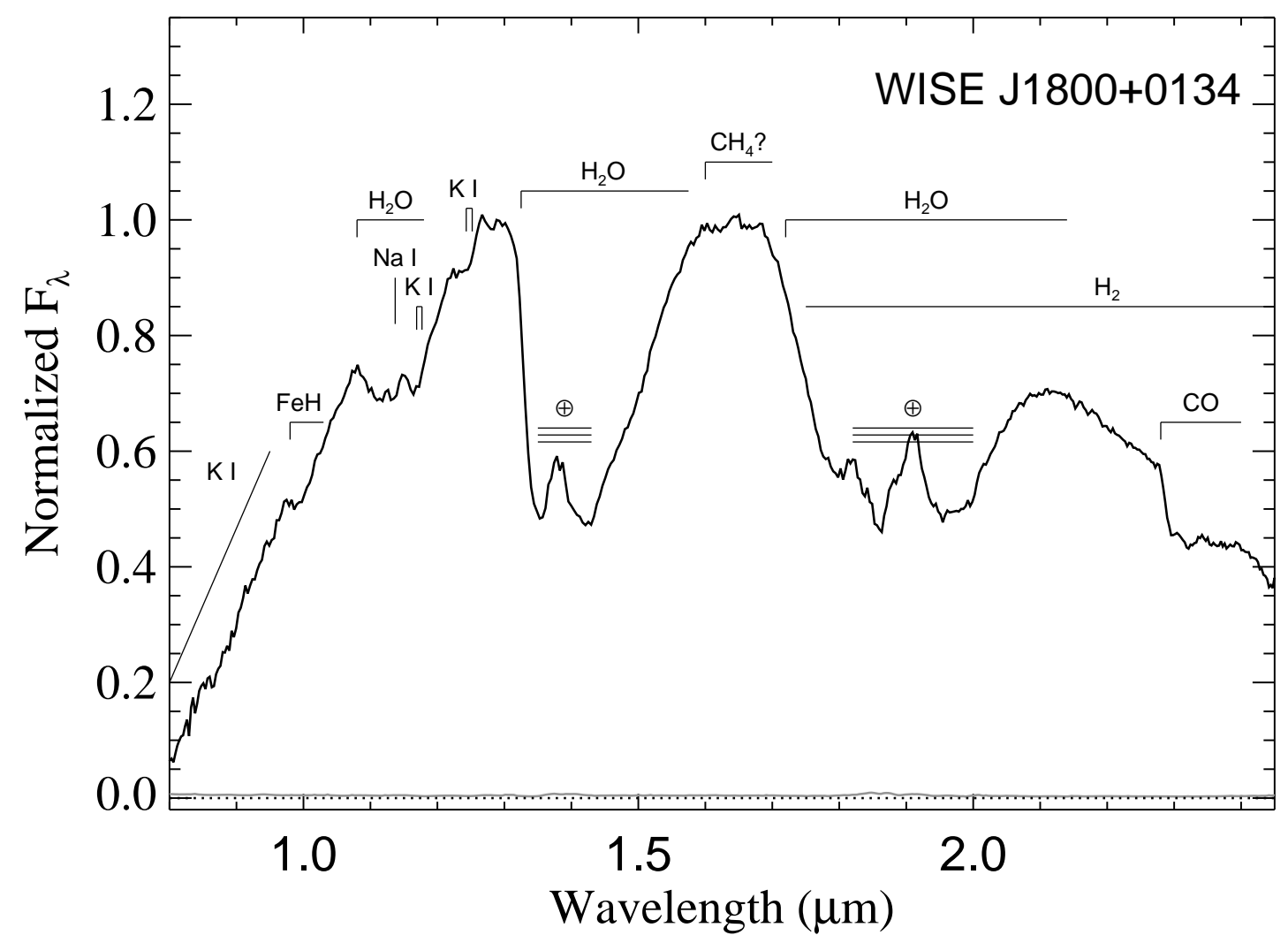

Fig. 2.- Low-resolution near-infrared spectrum of W1800+0134 obtained with SpeX. Data are normalized at $1.27 \mu \mathrm{m}$, and the uncertainty spectrum is shown in gray. Prominent atomic and molecular features are labeled. The $\mathrm{CH}_{4}$ region at $1.6 \mu$ mshows some flattening, but fits to the spectrum including an unresolved $\mathrm{T}$ dwarf companion are not statistically robust. 